\title{
Evaluation of clinical and inflammatory profile in opioid addiction patients with comorbid pain: results from a multicenter investigation
}

This article was published in the following Dove Press journal:

Neuropsychiatric Disease and Treatment

19 November 2014

Number of times this article has been viewed

\author{
Brittany B Dennis' \\ M Constantine Samaan ${ }^{2}$ \\ Monica Bawor ${ }^{3}$ \\ James Paul ${ }^{4}$ \\ Carolyn Plater ${ }^{5}$ \\ Guillaume Pare' \\ Andrew Worster ${ }^{6}$ \\ Michael Varenbut ${ }^{5}$ \\ Jeff Daiter ${ }^{5}$ \\ David C Marsh ${ }^{5,7}$ \\ Dipika Desai ${ }^{8}$ \\ Lehana Thabane ${ }^{1,9,10}$ \\ Zainab Samaan ${ }^{1,8,11}$
}

'Department of Clinical Epidemiology and Biostatistics, ${ }^{2}$ Department of Pediatrics, Division of Pediatric Endocrinology, ${ }^{3}$ McMaster Integrative Neuroscience Discovery and Study Program, ${ }^{4}$ Department of Anesthesia, McMaster University, Hamilton,

${ }^{5}$ Ontario Addiction Treatment Centres, Richmond Hill, ${ }^{6}$ Department of Medicine, Hamilton General Hospital, Hamilton, ${ }^{7}$ Northern Ontario School of Medicine, Sudbury, ${ }^{8}$ Population Genomics Program, Chanchlani Research Centre, McMaster University, Hamilton, ${ }^{9}$ Centre for Evaluation of Medicine, ${ }^{10}$ System Linked Research Unit, Hamilton, "Department of Psychiatry and Behavioural Neurosciences, McMaster University, Hamilton, ON, Canada

Correspondence: Zainab Samaan Department of Psychiatry and Behavioural Neurosciences, McMaster University, 1280 Main Street West, Hamilton, ON, Canada L8S 4L8

Tel + I 905522 II 55 (ext 36372)

Fax + I 9055756029

Email samaanz@mcmaster.ca
Background: Chronic pain is the most commonly reported comorbidity among patients with opioid addiction receiving methadone maintenance treatment (MMT), with an estimated prevalence ranging between $30 \%$ and 55\%. Evidence suggests that patients with comorbid pain are at high risk for poor treatment response, including continued illicit substance use. Due to the important relationship between the presence of pain and illicit substance abuse within the MMT setting, it is imperative that we target our efforts toward understanding the characteristics of this patient population.

Methods: The primary objective of this study was to explore the clinical and inflammatory profile of MMT patients reporting comorbid pain. This multicenter study enrolled patients ( $\mathrm{n}=235$ ) on MMT for the treatment of opioid dependence. Clinical history and blood and urine data were collected. Blood samples were obtained for estimating the serum levels of inflammatory markers (tumor necrosis factor [TNF]- $\alpha$, interleukin-1 receptor antagonist [IL-1ra], IL-6, IL-8, IL-10, interferon [IFN]- $\gamma$ and chemokine (C-C motif) ligand 2 [CCL2]). The study objectives were addressed using a descriptive statistical summary and a multivariable logistic regression model constructed in STATA version 12.

Results: Among the participants eligible for inclusion $(n=235)$, serum IFN- $\gamma$ level and substance abuse behavior proved to be important delineating characteristics for the detection of comorbid pain. Analysis of inflammatory profile showed IFN- $\gamma$ to be significantly elevated among patients reporting comorbid pain (odds ratio [OR]: $2.02 ; 95 \%$ confidence interval [CI]: $1.17,3.50 ; P=0.01$ ). Patients reporting comorbid pain were also found to have an increase in positive opioid urine screens (OR: 1.02; 95\% CI: 1.00, 1.03; $P=0.01$ ), indicating an increase in illicit opioid consumption.

Conclusion: MMT patients with comorbid pain were shown to have elevated IFN- $\gamma$ and higher rates of continued opioid abuse. The ability to objectively distinguish between patients with comorbid pain may help to both improve the prediction of poor responders to MMT as well as identify treatment approaches such as anti-inflammatory medications as safe alternatives for MMT patients with comorbid pain.

Keywords: methadone maintenance treatment, inflammatory markers, TNF- $\alpha$, IFN- $\gamma$, interleukins, CCL2, Brief Pain Inventory, opioid dependence

\section{Introduction}

Attention toward improving treatments for opioid dependence is increasing in conjunction with efforts to control the abuse of opioids. These efforts are seriously challenged by the increase in opioid prescriptions worldwide, and the global population of opioid users is now estimated to be 21.9 million people. ${ }^{1}$ Methadone - a synthetic opioid - is the most common treatment for opioid dependence. ${ }^{2}$ It is given to alleviate 
the symptoms of withdrawal and prevent relapse. ${ }^{2}$ Studies examining patients on methadone maintenance treatment (MMT) report chronic pain as a common comorbid disorder, with prevalence ranging from as low as $37 \%$ in some studies ${ }^{3}$ to as high as $55.3 \%$ in others. ${ }^{4}$ Chronic pain is both prevalent and concerning for patients with opioid addiction. Patients with comorbid chronic pain report a higher incidence of continued opioid abuse (COA). ${ }^{3,5,6}$ Concomitant use of illicit opioids in combination with MMT poses a serious risk of abnormal cardiac conductivity, ${ }^{7,8}$ overdose, ${ }^{9,10}$ and death. ${ }^{9}$ MMT patients with comorbid chronic pain are thought to be in the highest risk category for such adverse events due to the larger amount of illicit opioid consumption that chronic pain patients report. ${ }^{3,5,6}$ Such reported outcomes, in combination with the high reported prevalence of pain, dictate the need for further investigation into the characteristics and treatment effects of pain in patients with opioid use disorder. Determining the important delineating features of pain among MMT patients will help clinicians to develop a stronger understanding of the clinical profile and risks associated with comorbid pain.

Inflammatory profile is a recent development in the search for objective measures of pain and serves as a possible source of discrimination between patients with and without chronic pain. Both cytokines and chemokines operate as neuromodulators, regulating neuroinflammation and neurodevelopment. ${ }^{11}$ The deregulation of cytokines and chemokines is associated with both neuroinflammation and neurodegeneration, ${ }^{12,13}$ and any increase in neuroinflammation can result in neuropathic pain as well as inflammation. ${ }^{14-16}$ Proinflammatory cytokines and chemokines have been noted to also provoke hyperalgesia. ${ }^{17,18}$ One such study demonstrated a dose-response relationship between elevated cytokine levels (interleukin [IL]-1 $\beta$, IL-2, IL-6, interferon [IFN]- $\gamma$, and tumor necrosis factor [TNF]- $\alpha$ ) and chronic pain severity. ${ }^{18}$ However, this study was restricted by a small sample size (94 patients with pain and six healthy controls), wherein most cytokines failed to reach significance after adjusting for multiple testing. ${ }^{18}$

Due to the important relationship between the presence of pain and illicit substance abuse, as well as the overwhelming presence of pain within the methadone setting, it is imperative that we target our efforts toward understanding the characteristics of this patient population. Understanding pain is not only important in preventing adverse health outcomes for patients, it is vital for reducing social expenditure on treatments that may stand ineffective for specific subpopulations. The studies examining the characteristics of chronic pain are small in number and marked by inconsistent findings. There are an equal number of studies reporting a positive association between chronic pain and $\mathrm{COA}^{3,5}$ as those reporting no significant findings. ${ }^{19,20}$ Moreover, we have yet to properly identify the mechanisms of pain among MMT patients. These shortcomings prompted us to commence a sizable investigation of MMT patients to address our primary research objective, namely, to explore the clinical and inflammatory profile of MMT patients reporting comorbid pain. We addressed our objective using data collected for the GENetics of Opioid Addiction (GENOA) research collaborative. ${ }^{21}$ GENOA is a multicenter cross-sectional investigation, accomplished through the partnership between McMaster University and the Ontario Addiction Treatment Centres (OATC). ${ }^{21}$

\section{Methods \\ Overview of GENOA}

Data have been collected for this study from the GENOA research collaborative between the OATC - the largest MMT network of opioid dependence treatment centers in North America - and the Population Genomics Program in the Faculty of Health Sciences at McMaster University. The detailed methodology of the GENOA investigation has been described previously. ${ }^{21}$ The GENOA study is a multicenter cross-sectional analysis, which includes clinical data from four sites (methadone clinics) in southern Ontario. Participants were enrolled in the study between June and December 2011. The Hamilton Integrated Research Ethics Board approved this study.

The study inclusion criteria were as follows: men and women, age $\geq 18$ years, ability to provide informed consent and willingness to provide a blood sample and receiving methadone for opioid-dependence treatment. All study participants were diagnosed with opioid dependence according to Diagnostic and Statistical Manual of Mental Disorders, Fourth Edition criteria, based on clinical interviews at the time of entry into treatment with methadone. This study will focus on the data collected from $235 \mathrm{MMT}$ patients (Figure 1), investigating the relationship between self-reported comorbidities and methadone response. Information on participants' physical comorbidities was gathered from face-to-face clinical interviews performed by trained OATC nurses. The presence of chronic and/or comorbid pain was determined by asking patients to respond to the following question: "Are you currently experiencing or have been diagnosed with chronic pain?" The use of this question to define chronic pain cases has been validated against the Brief Pain Inventory (BPI) in a previous study (Dennis et al, unpublished data, 2014). 
Preliminary screening for GENOA:

men and women, age $\geq 18$ years who are able to provide

written informed consent, on substitute opioid therapy for

the treatment of opioid addiction

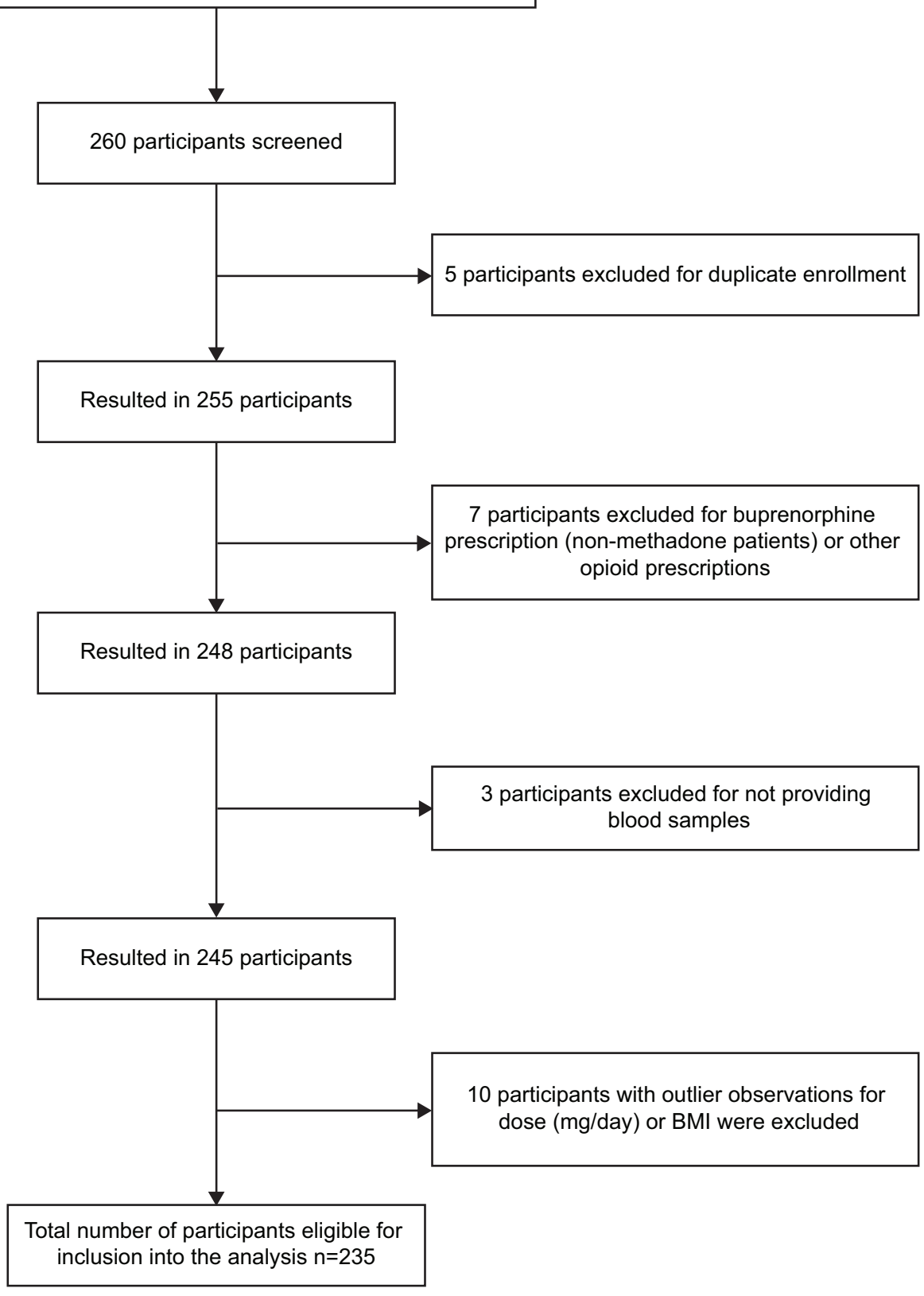

Figure I Flow diagram for eligibility screening and participant selection.

Abbreviations: GENOA, GENetics of Opioid Addiction; BMI, body mass index.

Results from the validation suggest that simply asking patients whether they have pain shows an $88.8 \%$ specificity, $84.4 \%$ positive predictive value, and C-statistic of 0.69 . COA was determined through the assessment of weekly urinalysis for illicit opioid testing. Pain was also examined in relationship to the following inflammatory markers: TNF- $\alpha$, IL-1 receptor antagonist (IL-1 ra), IL-6, IL-8, IL-10, IFN- $\gamma$, and chemokine
(C-C motif) ligand 2 (CCL2) in serum. Evidence shows that different anticoagulants (such as ethylene diamine tetraacetic acid, present in blood collection tubes) influence absolute cytokine levels in various manners ${ }^{22-24}$ because serum samples were used in preference to plasma.

Interviewers obtained weight and height measurements from all participants. Information on social demographic 
factors, medical history, methadone dose, methadone treatment duration, family history of drug use, and psychiatric disorders were obtained during the interview process. All participants received the Mini International Neuropsychiatric Interview drug and alcohol modules. Blood samples were taken for estimation of serum levels of inflammatory markers. Participant blood specimens were processed within 2 hours and stored on site in $-20^{\circ} \mathrm{C}$ freezers, then shipped monthly to the Hamilton research laboratory, and stored in liquid nitrogen until the time of analysis.

\section{Laboratory analyses}

Laboratory measures included urine toxicology screens to measure illicit opioid abuse and Bio-Plex ${ }^{\mathrm{TM}}$ (Bio-Rad Laboratories, Hercules, CA, USA) cytokine assay ${ }^{25}$ to measure serum inflammatory markers.

\section{Urine analysis}

Qualitative and semiquantitative urinalysis was conducted using $\mathrm{iMDx}^{\mathrm{TM}}$ Prep assay. ${ }^{26}$ The $\mathrm{iMDx}{ }^{\mathrm{TM}}$ Prep assays are intended for the measurement of drugs of abuse, as well as the identification of adulteration in human urine samples, on the $\mathrm{iMDx}^{\mathrm{TM}}$ Analyzer and are used in drug rehabilitation clinics and physician offices by trained users. OATC clinics require patients to provide weekly urine samples as part of routine clinical care. While participants are also tested for cocaine, tetrahydrocannabinol, and benzodiazepines, we are primarily interested in the patients' use of opioids. Using the iMDx ${ }^{\mathrm{TM}}$ Prep assays, we are able to differentiate between specific types of opioids, such as naturally occurring opioids (heroin), prescribed synthetic opioids, and methadone. ${ }^{26}$ In this investigation, opioid use is an indicator for methadone response. Because methadone is not used for the treatment of benzodiazepine or cocaine addiction, a patient's continued use of these substances does not indicate a methadone treatment failure. Urine toxicology screening was used to determine whether opioids (natural and synthetic) were present in the participants' urine.

Participants provided urine samples at supervised facilities; there were no missing urine samples from study participants. COA was determined by calculating the percentage of positive opioid urine screens provided by participants (number of positive opioid urine screens/total number of opioid urine screens). High COA percentage is indicative of a high number of positive opioid urine screens or, alternatively, a higher rate of illicit opioid consumption. We chose to include a measure of continued opioid abuse that adjusts for the entire duration of methadone treatment. Opioid dependence is a remitting, relapsing disorder, and as such, restricting the measurement of response to such a short time frame of the patient's overall treatment course is of limited use.

\section{Serum levels of inflammatory markers: Bio-Plex assay}

Serum samples were collected from participants using BD Vacutainer tubes and allowed to clot for 30 minutes. Samples were centrifuged at $1,500 \times g$ for 15 minutes at room temperature and the serum was frozen in liquid nitrogen until further analysis.

Samples were thawed only once and $50 \mu \mathrm{L}$ aliquots were transferred to 96-well plates. Serum cytokine levels were determined using the Bio-Plex assay (Bio-Rad Laboratories); levels of IL-6, IL-8, IL-1ra, TNF- $\alpha$, IFN- $\gamma$, IL-10, IL-1 $\beta$, and CCL2 were measured, and standard curves were generated as per manufacturer's instructions. The Bio-Plex Manager 6.0 software was used for data analysis. Cytokine measurements were expressed as picograms per milliliter.

While IL-1B was originally tested for in all participants, more than $50 \%$ of the samples were inconclusive. With such a high proportion of data missing, we chose not to include IL-1B in any analyses.

\section{Statistical analysis}

STATA version 12 was used to complete all analyses. All study data have been quality checked and entered into the Research Electronic Data Capture database at the Population Genomics Program, McMaster University.

Multiple imputation using chained equations was employed to adjust for missing data. Age, sex, COA, chronic pain, and methadone dose (milligrams per day) were the variables selected to aid in the multiple imputation prediction of missing values. When running analyses of inflammatory biomarkers, if the value was below detectable range, the lowest value before detection cutoff was imputed. All data were tested for normal distribution, where log transformations were made when necessary. All outlier data were removed before performing the primary analyses. To adjust for outlier variables, box plots were constructed for all predictors included in each model using STATA version 12, these being methadone dose, duration on MMT, age, body mass index, and all inflammatory biomarkers. The box plots resulted in the identification of ten outlier observations across predictors $\left(\mathrm{n}_{\text {participants }}=10\right)$. The inflammatory biomarkers proved to have an overwhelming number of outlier observations due to their wide distribution, limiting our ability to adequately remove them from the sample (Figure 2). However, we 


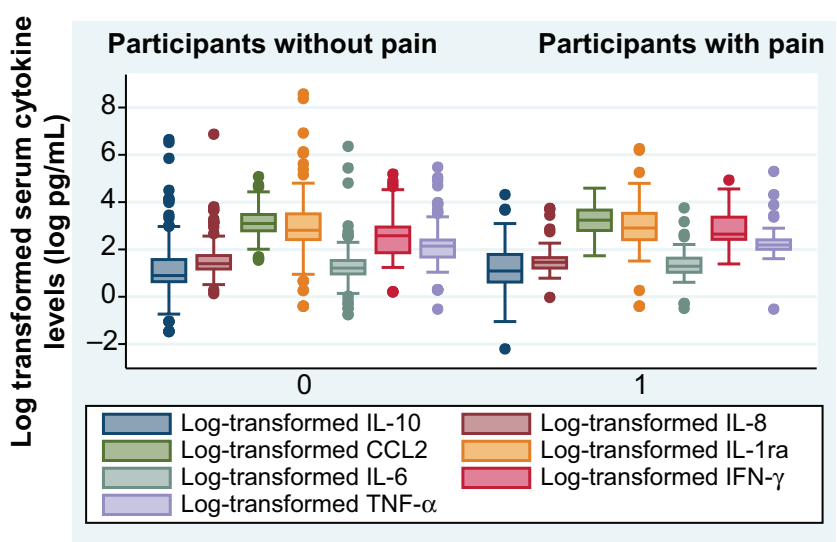

Figure 2 Distribution of inflammatory biomarkers.

Notes: Cytokine data provided in this figure were originally measured in picograms per milliliter using participants' serum samples; the distribution here is provided using log-transformed values.

Abbreviations: IL, interleukin; IL-I ra, interleukin I receptor antagonist; TNF, tumor necrosis factor; $\mathrm{CCL}$, chemokine ( $\mathrm{C}-\mathrm{C}$ motif) ligand 2; IFN, interferon.

acknowledge how sensitive inflammatory profiles are and that currently no normal range has been established in the MMT patient population.

We determined the appropriateness of our sample size $(n=235)$ to address our primary analysis, the multivariable logistic regression of chronic pain. With response to treatment (COA) as our primary independent variable, in addition to eleven other a priori defined covariates, we determined that our model could withstand the addition of 20 covariates under the assumption that model stability is maintained with ten to 12 observations per covariate. Within this model, we have added 12 covariates, allowing for 20 observations per covariate in our sample of $235 .{ }^{27}$ Reporting of this study follows the Strengthening of Reporting of Observational Studies in Epidemiology (STROBE) guidelines. ${ }^{28}$

\section{Primary analysis}

All demographic characteristics are summarized using descriptive statistics, reporting means and standard deviations (SDs) for continuous values and percentages for dichotomous values. All demographic characteristic data are presented by pain status. A multivariable logistic regression model was constructed to address our primary objective, determining the clinical and inflammatory profile of patients reporting comorbid pain, where self-reported pain was the binary dependent variable. This model included multiple covariates identified as or trending toward significance during the univariate analysis (age, IFN- $\gamma$, and response to treatment [COA]). The model also adjusted for important confounding variables, such as age, presence of inflammatory medications, sex, presence of infectious disease, and methadone dose (milligrams per day).

\section{Results}

\section{Demographic characteristics of GENOA participants}

The recruitment process led to a completed sample of 249 participants eligible for this study. Any participants reporting prescribed opioids in their current medication list were removed from any analyses, leaving us with a sample of 235 MMT patients. A flow diagram of participant screening and selection is presented in Figure 1.

Among the participants eligible for inclusion into the analyses $(n=235), 40.42 \%$ were female, with mean age of 36.82 (SD: 10.36) years and mean body mass index of 26.59 (SD: 5.46$) \mathrm{kg} / \mathrm{m}^{2}$. Participants self-reported the following comorbidities: $0.43 \%$ human immunodeficiency virus infection, $22.98 \%$ hepatitis, 5.11\% liver disease, $24.68 \%$ chronic pain, $2.13 \%$ epilepsy, and $23.40 \%$ other, with a total of $58.40 \%$ of participants reporting at least one of the aforementioned comorbidities. When asked to indicate any "other" physical comorbidities, participants' responses included the following: diabetes $(n=8)$, cardiac functioning abnormalities and stroke history $(n=7)$, hypertension $(n=3)$, high cholesterol $(n=1)$, neurological deficit $(n=2)$, Crohn's disease $(n=4)$, asthma $(n=8)$, renal functioning problems $(n=2)$, gall stones $(n=3)$, fibromyalgia $(n=1)$, thyroid abnormalities $(n=3)$, arthritis $(n=5)$, respiratory problems $(n=2)$, allergies $(n=3)$, hernia $(n=1)$, gout $(n=1)$, spondylitis $(n=1)$, and endometriosis $(n=1)$. Reporting of these "other" comorbidities did not vary between patients with and without pain. All participants' demographic information presented by pain status is summarized in Table 1.

\section{Clinical and inflammatory profile of MMT patients with comorbid pain}

The demographic characteristics summarized in Table 1 suggest that participants reporting pain are similar in demographic and clinical profiles to participants without pain. We find age, methadone dose (milligrams per day), sex, treatment duration (months), and onset age of opioid abuse to be relatively the same across patient groups (Table 1). A distinct aspect of the clinical profile for patients with pain is noted in the significantly different treatment response rates across groups. Another distinction between patients with and without pain is their inflammatory profile, whereby we found participants with pain to have elevated IFN- $\gamma$, trending toward significance. 
Table I Participant demographic characteristics (divided by pain status) ( $\mathrm{n}=235)$

\begin{tabular}{|c|c|c|c|}
\hline & $\begin{array}{l}\text { Comorbid } \\
\text { pain }(n=58)\end{array}$ & $\begin{array}{l}\text { No comorbid } \\
\text { pain }(n=\mid 77)\end{array}$ & $\begin{array}{l}P \text {-value } \\
\text { (univariate } \\
\text { analysis) }\end{array}$ \\
\hline \multicolumn{4}{|l|}{ Demographic characteristics } \\
\hline Female (\%) & 41.38 & 40.11 & 0.90 \\
\hline Mean age (years) & $39.45( \pm 10.29)$ & $35.95( \pm 10.26)$ & 0.02 \\
\hline Mean BMI $\left(\mathrm{kg} / \mathrm{m}^{2}\right)$ & $27.46( \pm 5.08)$ & $26.31( \pm 5.56)$ & 0.15 \\
\hline Mean methadone dose $(\mathrm{mg} / \mathrm{d})$ & $84.64( \pm 5 I .5 I)$ & $85.74( \pm 50.14)$ & 0.76 \\
\hline $\begin{array}{l}\text { Mean response to MMT (mean \% opioid-positive } \\
\text { urine screens) }\end{array}$ & $23.99( \pm 27.14)$ & $15.82( \pm 20.11)$ & 0.02 \\
\hline Duration on MMT (months) & $41.31( \pm 38.99)$ & $38.25( \pm 42.79)$ & 0.61 \\
\hline Mean onset age of opioid abuse & $23.21( \pm 11.28)$ & $23.16( \pm 8.61)$ & 0.98 \\
\hline Patients with HIV (\%) & 0.00 & 0.56 & Unable to determine \\
\hline Patients with hepatitis (\%) & 29.31 & 20.90 & 0.22 \\
\hline \multicolumn{4}{|l|}{ Inflammatory profile } \\
\hline IL-10 & $1.15( \pm I .14)$ & $1.16( \pm 1.28)$ & 0.86 \\
\hline IL-8 & $1.55( \pm 0.67)$ & $1.56( \pm 0.76)$ & 0.97 \\
\hline CCL2 & $3.25( \pm 0.60)$ & $3.14( \pm 0.57)$ & 0.26 \\
\hline IL-I ra & $2.96( \pm 1.30)$ & $2.96( \pm I .33)$ & 0.92 \\
\hline IL-6 & $1.35( \pm 0.72)$ & $1.30( \pm 0.85)$ & 0.62 \\
\hline IFN- $\gamma$ & $2.78( \pm 0.89)$ & $2.55( \pm 0.89)$ & 0.08 \\
\hline TNF- $\alpha$ & $2.25( \pm 0.77)$ & $2.20( \pm 0.80)$ & 0.69 \\
\hline
\end{tabular}

Notes: All inflammatory biomarker concentrations have been log-transformed for this table (originally measured as picograms per milliliter). These are the results for the 235 participants eligible for study inclusion; outliers identified for BMI and methadone dose were removed for regression models ( $\mathrm{n}=\mathrm{I0}$ ). Data are presented as mean ( \pm standard deviation).

Abbreviations: MMT, methadone maintenance treatment; IL, interleukin; IL-Ira, interleukin I receptor antagonist; BMI, body mass index; TNF, tumor necrosis factor; CCL2, chemokine (C-C motif) ligand 2; HIV, human immunodeficiency virus; IFN, interferon.

We chose to construct a multivariable logistic regression model to further assess these associations using patientreported pain as our outcome of interest. Regression models allow the assessment of association between factors while also adjusting for other important confounders. Using results from the univariate analysis to guide our selection of covariates, we included COA (treatment response) and IFN- $\gamma$ as our primary independent variables. We adjusted this model for presence of inflammatory medications, sex, presence of infectious disease, and methadone dose (milligrams per day). The results from the multivariable regression model are summarized in Table 2. Results suggested IFN- $\gamma$ to be significantly elevated among patients reporting chronic pain, while adjusting for important covariates (odds ratio [OR]: $2.02 ; 95 \%$ confidence interval $[\mathrm{CI}]: 1.17,3.50 ; P=0.01)$. The results also suggest that patients reporting comorbid pain

Table 2 Clinical and inflammatory characteristics of comorbid pain: a multivariable logistic regression model $(n=235)$

\begin{tabular}{llll}
\hline Covariates & Odds ratio & 95\% Confidence interval & P-value \\
\hline Age (years) & 1.03 & $0.99,1.06$ & 0.08 \\
Sex & 1.08 & $0.56,2.07$ & 0.82 \\
Response to MMT (\% positive opioid urine tests) & 1.02 & $1.00,1.03$ & 0.01 \\
Infectious disease status & 1.40 & $0.65,3.00$ & 0.38 \\
Methadone dose (mg/d) & 1.00 & $0.99,1.01$ & 0.94 \\
Presence of inflammatory medications & 1.26 & $0.41,3.92$ & 0.69 \\
TNF- $\alpha$ & 0.69 & $0.37,1.30$ & 0.25 \\
IFN- $\gamma$ & 2.02 & $1.17,3.50$ & 0.01 \\
IL-6 & 1.18 & $0.60,2.32$ & 0.63 \\
IL-Ira & 0.84 & $0.5 I, 1.37$ & 0.49 \\
CCL2 & 1.60 & $0.88,2.88$ & 0.12 \\
IL-8 & 0.73 & $0.43,1.21$ & 0.22 \\
IL-I0 & 1.01 & $0.69,1.48$ & 0.97 \\
\hline Note & &
\end{tabular}

Notes: Sex is interpreted as female, in reference to males. Infectious disease status was a binary measure of the presence of HIV and/or hepatitis. All cytokine measurements have been log-transformed, and the original measurements were in picograms per milliliter.

Abbreviations: MMT, methadone maintenance treatment; IL, interleukin; IL-I ra, interleukin I receptor antagonist; TNF, tumor necrosis factor; CCL2, chemokine (C-C motif) ligand 2; HIV, human immunodeficiency virus; IFN, interferon. 
have an increase in positive opioid urine screens (OR: 1.02; $95 \%$ CI: $1.00,1.03 ; P=0.01)$, indicating an increase in illicit opioid consumption.

\section{Discussion}

\section{Summary of findings}

Considerations of pain in the clinical setting for patients on MMT for opioid dependence are complicated by the inconsistent findings reported across studies. While some studies appear to be reporting a strong association between chronic pain and substance abuse among MMT patients, ${ }^{3,5}$ other studies report no association. ${ }^{19,20}$ There is also limited research on the inflammatory characteristics of pain patients within the MMT setting. Results from this investigation provide a thorough evaluation of the clinical and inflammatory characteristics of opioid-dependent patients with pain, wherein we show that 1) response to MMT is significantly influenced by the presence of pain and 2) MMT patients reporting chronic pain show elevated levels of IFN- $\gamma$.

\section{Context of comorbid pain and opioid abuse in the current literature}

MMT patients with severe pain are known to have increased methadone dose $\mathrm{e}^{4}$ and an increased rate of illicit substance use. ${ }^{4}$ Findings from this study are consistent with some of those in literature, ${ }^{3,5}$ where response to treatment was highly associated with chronic pain status. When determining the source of contention across studies examining pain and opioid abuse, we took a closer look at the differences in measurement and definition of response to MMT. While in this study we chose to use the percentage of opioid-positive urine screens as an objective proxy outcome measure for response to methadone treatment, other studies report response to treatment as the number of days of illicit heroin or opioid abuse in the previous month ${ }^{5,20}$ or the percentage of patients who report using illicit opioids in the month. ${ }^{3,6,19}$ In addition, a number of studies rely on different measurements for response such as self-report, ${ }^{3,6,19}$ and some studies go so far as using validated tools to assess the severity of substance abuse behavior. ${ }^{5,20}$

In comparison to our investigation, the majority of clinical studies assess response to treatment over a very short time frame ( 7 days to 3 months)., ${ }^{3,20,29}$ It is known that opioid dependence is a chronic, remitting, relapsing disorder, with the average methadone treatment duration being 2 years. As such, capturing "response" over a short time frame of a patient's overall treatment course appears of limited use. Determining response to MMT by reviewing patients for the entire duration of MMT appeals as a more adequate approach for characterizing the course of and patient response to methadone. In this study, we looked at the number of positive opioid urine screens as a percentage of the total number of screens in an effort to adjust for these duration effects, which may explain why our results may differ from studies basing treatment response on a shorter time frame (ie, 7-9 days). ${ }^{20}$

Similar to the measurement of response, the measurement of chronic pain also varies across studies. This variation may also be a source of discrepancy in the reported findings in the current literature. The measurement of pain varies from validated pain measures in some studies ${ }^{3,20}$ to the use of self-reported pain in others. ${ }^{6}{ }^{19}$ Even results from studies selecting "validated" pain measures such as the $\mathrm{BPI}^{3}$ should be interpreted with caution, for no pain measurement tool has undergone specific psychometric testing or predictive/ criterion validation within the MMT patient population.

\section{Inflammatory profile and comorbid pain}

Our results have shown IFN- $\gamma$ to be elevated among MMT patients reporting comorbid pain. The role of IFN- $\gamma$ in pain can be inferred from animal studies in which IFN- $\gamma$ is noted to induce pain. ${ }^{30}$ Tsuda et al ${ }^{30}$ found that the IFN- $\gamma$ receptor mediates spinal microgilia activation, ultimately leading to neuropathic pain. ${ }^{30}$ When the spinal microglia is activated, it increases pain processing inside the dorsal horn to a significant level that triggers neuropathic pain. ${ }^{31-34}$ This is one of the mechanisms by which inflammation causes and propagates pain.

Our findings are consistent with other studies, where IFN- $\gamma$ is elevated during periods of pain. ${ }^{18,35}$ In one investigation, 21 patients with lumbar degenerative disc disease were compared against three controls, for inflammatory profile differences, where the authors identified immunoreactivity of IFN- $\gamma$ in patients with axial back pain..$^{35}$ Another study, examining 94 chronic pain patients and six healthy volunteers, found that proinflammatory cytokines such as IL- $1 \beta$, IL-2, IL-6, IFN- $\gamma$, and TNF- $\alpha$ correlated with increasing pain intensity. ${ }^{18}$ In addition, proinflammatory cytokines have been demonstrated to directly oppose opioid actions, and one study has demonstrated that an increase in morphine and methadone administration is directly linked to an increase in spinal glial activation as well as elevated cytokine level. ${ }^{36}$

To date, no study has explored the association between comorbid pain and inflammatory profile within the MMT patient population. This investigation showed the significant association between elevated IFN- $\gamma$ level and the presence of chronic pain. The importance of these results rests on 
our understanding of treatment strategies for patients with concurrent opioid dependence and chronic pain. The ability to objectively distinguish between patients with comorbid pain through the identification of IFN- $\gamma$ may be able to help distinguish treatment approaches such as anti-inflammatory medications as a safe alternative to opioid analgesics in this patient population.

\section{Strengths and limitations}

The major limitation of this study is the use of self-reported chronic pain. The true prevalence of pain could have been under- or overestimated. Without the use of a validated pain assessment for opioid-dependent patients receiving MMT, the reported results should be subject to cautious interpretation. However, in a recent study, we have validated the use of patient-reported pain in comparison with the BPI assessment, where results suggest that simply asking patients whether they have pain shows an $88.8 \%$ specificity, $84.4 \%$ positive predictive value, and C-statistic of 0.69 . Such results indicate that the use of patient-reported pain very closely identifies the same population as the BPI assessment. In addition, we should not discount the use of more objective markers for reported pain. This study found elevated levels of inflammatory markers, supporting the case for both the use of objective pain indicators and consideration of anti-inflammatory agents as adjunct therapy for MMT patients.

\section{Conclusion}

While our study shows a significant association between pain and poor response to MMT, it also proves the importance of determining an objective measure of inflammation for MMT patients with comorbid pain. We determined that pain is significantly associated with an increase in positive opioid urine screens, as well as a substantial elevation of IFN- $\gamma$. In an effort to adequately manage patients at an increased risk for methadone overdose and poor response, future research should determine the therapeutic impact of using antiinflammatory analgesics to prevent the use of illicit opioids and reduce pain in opioid-dependent patients on MMT.

\section{Acknowledgments}

We sincerely thank everyone who contributed to the completion of this project. This project would not have been possible without the great collaboration cemented between GENOA and the OATC network of clinical sites. We extend our sincere appreciation to all the participants from the OATC facilities who generously donated their time, information, and samples, without which this study would not be possible.
We also extend our gratitude to the OATC clinical staff for all their great efforts in patient recruitment and data collection. We thank Jacqueline Hudson for her great dedication as the research coordinator on the GENOA team. We also thank the McMaster University undergraduate students, including Sindoora Iyer, Leen Naji, Anuja Bhalerao, Herman Bami, and Andrew Kamphuis, who spent a great deal of time in helping with data entry and cleaning.

This work was supported by a grant from the Drug Safety and Effectiveness Network of the Canadian Institutes of Health Research (number 126639). The funders had no role in design and conduct of the study; collection, management, analysis, and interpretation of the data; and preparation, review, or approval of the manuscript.

\section{Author contributions}

Dr Zainab Samaan, Brittany B Dennis, and Dr Lehana Thabane were responsible for the development of the question and research protocol for this study. Brittany B Dennis was responsible for all statistical analyses performed in this investigation. Dr M Constantine Samaan designed and performed all laboratory analyses for the inflammatory profile. All authors contributed equally during manuscript development. Zainab Samaan had full access to data from this investigation, and she is accountable for the reliability of the data and the accuracy of all analyses performed. All authors contributed toward data analysis, drafting and revising the paper and agree to be accountable for all aspects of the work.

\section{Disclosure}

The authors report no conflicts of interest in this work.

\section{References}

1. UNODC. World Drug Report; 2010. Available from: http://www.unodc. org/unodc/en/data-and-analysis/WDR-2010.html. Accessed: September $2,2014$.

2. Effective medical treatment of opiate addiction. National consensus development panel on effective medical treatment of opiate addiction. JAMA. 1998;280(22):1936-1943.

3. Rosenblum A, Joseph H, Fong C, Kipnis S, Cleland C, Portenoy RK. Prevalence and characteristics of chronic pain among chemically dependent patients in methadone maintenance and residential treatment facilities. JAMA. 2003;289(18):2370-2378.

4. Peles E, Schreiber S, Gordon J, Adelson M. Significantly higher methadone dose for methadone maintenance treatment (MMT) patients with chronic pain. Pain. 2005;113(3):340-346.

5. Trafton JA, Oliva EM, Horst DA, Minkel JD, Humphreys K. Treatment needs associated with pain in substance use disorder patients: implications for concurrent treatment. Drug Alcohol Depend. 2004;73(1):23-31.

6. Barry DT, Bernard MJ, Beitel M, Moore BA, Kerns RD, Schottenfeld RS. Counselors' experiences treating methadone-maintained patients with chronic pain: a needs assessment study. J Addict Med. 2008;2(2): 108-111. 
7. Peles E, Bodner G, Kreek MJ, Rados V, Adelson M. Corrected-QT intervals as related to methadone dose and serum level in methadone maintenance treatment (MMT) patients: a cross-sectional study. Addiction. 2007;102(2):289-300.

8. Krantz MJ, Kutinsky IB, Robertson AD, Mehler PS. Dose-related effects of methadone on QT prolongation in a series of patients with torsade de pointes. Pharmacotherapy. 2003;23(6):802-805.

9. Cao X, Wu Z, Li L, et al. Mortality among methadone maintenance clients in China: a six-year cohort study. PLoS One. 2013;8(12):e82476.

10. Bohnert AS, Ilgen MA, Trafton JA, et al. Trends and regional variation in opioid overdose mortality among veterans health administration patients, Fiscal Year 2001 to 2009. Clin J Pain. 2014;30(7):605-612.

11. Ramesh G, MacLean AG, Philipp MT. Cytokines and chemokines at the crossroads of neuroinflammation, neurodegeneration, and neuropathic pain. Mediators Inflamm. 2013;2013:480739.

12. Smith JA, Das A, Ray SK, Banik NL. Role of pro-inflammatory cytokines released from microglia in neurodegenerative diseases. Brain Res Bull. 2012;87(1):10-20.

13. Glass CK, Saijo K, Winner B, Marchetto MC, Gage FH. Mechanisms underlying inflammation in neurodegeneration. Cell. 2010;140(6): 918-934.

14. Watkins LR, Maier SF. Beyond neurons: evidence that immune and glial cells contribute to pathological pain states. Physiol Rev. 2002;82(4): 981-1011.

15. Fallon BA, Levin ES, Schweitzer PJ, Hardesty D. Inflammation and central nervous system Lyme disease. Neurobiol Dis. 2010;37(3): 534-541.

16. Kiguchi N, Kobayashi Y, Kishioka S. Chemokines and cytokines in neuroinflammation leading to neuropathic pain. Curr Opin Pharmacol. 2012;12(1):55-61.

17. Gruber HE, Hoelscher GL, Ingram JA, Hanley EN Jr. Genome-wide analysis of pain-, nerve- and neurotrophin -related gene expression in the degenerating human annulus. Mol Pain. 2012;8(1):63.

18. Koch A, Zacharowski K, Boehm O, et al. Nitric oxide and proinflammatory cytokines correlate with pain intensity in chronic pain patients. Inflamm Res. 2007;56(1):32-37.

19. Barry DT, Beitel M, Joshi D, Schottenfeld RS. Pain and substancerelated pain-reduction behaviors among opioid dependent individuals seeking methadone maintenance treatment. Am J Addict. 2009;18(2): 117-121.

20. Ilgen MA, Trafton JA, Humphreys K. Response to methadone maintenance treatment of opiate dependent patients with and without significant pain. Drug Alcohol Depend. 2006;82(3):187-193.

21. Samaan Z, Bawor M, Dennis B, et al. Genetic influence in methadone dose and response to treatment in patients undergoing Methadone Maintenance Treatment (MMT) for opioid addiction: a pilot study. J Neuropsychiatr Dis Treat. 2014;10:1503-1508.
22. Marques-Vidal P, Bochud M, Bastardot F, et al. Levels and determinants of inflammatory biomarkers in a Swiss population-based sample (CoLaus study). PLoS One. 2011;6(6):e21002.

23. Flower L, Ahuja RH, Humphries SE, Mohamed-Ali V. Effects of sample handling on the stability of interleukin 6 , tumour necrosis factor-alpha and leptin. Cytokine. 2000;12(11):1712-1716.

24. Skeppholm M, Wallen NH, Blomback M, Kallner A. Can both EDTA and citrate plasma samples be used in measurements of fibrinogen and C-reactive protein concentrations? Clin Chem Lab Med. 2008;46(8): 1175-1179.

25. BIORAD Laboratories Inc. Bio-Plex ${ }^{\mathrm{TM}}$ Cytokine Assay. US patent 5,981,180; 6,046,807; 6,057,107. Available from http:/www.bio-rad. com/webroot/web/pdf/lsr/literature/BULLETIN_10008318A.pdf. Accessed October 24, 2014.

26. NOVA Systems. inventor; iMDx TM. Available from: http://www. novxsystems.com/novx/myweb.php?hls=10024. Accessed October 24, 2014.

27. Harrell, FE Jr. Regression Modeling Strategies: With Applications to Linear Models, Logistic Regression, and Survival Analysis. New York: Springer; 2001. [Corrected edition, January 10, 2001].

28. von Elm E, Altman DG, Egger M, Pocock SJ, Gotzsche PC, Vandenbroucke JP. Strengthening the Reporting of Observational Studies in Epidemiology (STROBE) statement: guidelines for reporting observational studies. BMJ. 2007;335(7624):806-808.

29. Barry D, Sullivan B, Petry NM. Comparable efficacy of contingency management for cocaine dependence among African American, Hispanic, and White methadone maintenance clients. Psychol Addict Behav. 2009;23(1):168-174.

30. Tsuda M, Masuda T, Kitano J, Shimoyama H, Tozaki-Saitoh H, Inoue $\mathrm{K}$. IFN-gamma receptor signaling mediates spinal microglia activation driving neuropathic pain. Proc Natl Acad Sci USA. 2009;106(19): 8032-8037.

31. Watkins LR, Milligan ED, Maier SF. Glial activation: a driving force for pathological pain. Trends Neurosci. 2001;24(8):450-455.

32. Tsuda M, Inoue K, Salter MW. Neuropathic pain and spinal microglia: a big problem from molecules in "small" glia. Trends Neurosci. 2005; 28(2):101-107.

33. Marchand F, Perretti M, McMahon SB. Role of the immune system in chronic pain. Nat Rev Neurosci. 2005;6(7):521-532.

34. Scholz J, Woolf CJ. The neuropathic pain triad: neurons, immune cells and glia. Nat Neurosci. 2007;10(11):1361-1368.

35. Cuellar JM, Golish SR, Reuter MW, et al. Cytokine evaluation in individuals with low back pain using discographic lavage. Spine J. 2010; 10(3):212-218.

36. Hutchinson MR, Coats BD, Lewis SS, et al. Proinflammatory cytokines oppose opioid-induced acute and chronic analgesia. Brain Behav Immun. 2008;22(8):1178-1189.
Neuropsychiatric Disease and Treatment

\section{Publish your work in this journal}

Neuropsychiatric Disease and Treatment is an international, peerreviewed journal of clinical therapeutics and pharmacology focusing on concise rapid reporting of clinical or pre-clinical studies on a range of neuropsychiatric and neurological disorders. This journa is indexed on PubMed Central, the 'PsycINFO' database and CAS,

\section{Dovepress}

and is the official journal of The International Neuropsychiatric Association (INA). The manuscript management system is completely online and includes a very quick and fair peer-review system, which is all easy to use. Visit http://www.dovepress.com/testimonials.php to read real quotes from published authors. 